\title{
A MODEL FOR FORCES ON TIRE-GROUND CONTACT REGION
}

\author{
Ronaldo de Breyne Salvagni \\ University of Sao Paulo, Escola Politecnica \\ E-mail: rbsalvagni@pobox.com
}

\begin{abstract}
The existing tire models are basically of three kinds: essentially empirical ("magic formulas"), or mixed empirical/analytical, or extremely complex theoretical models almost useless in practical situations. The model here proposed does not require any empirical data, and presents a simple theoretical approach very suitable to use in project and analysis of real suspension systems.

This paper presents a physical and mathematical model for some forces on tire-ground contact region of pneumatic car tires. It is a theoretical model, in the sense that it does not require any empirical data. It is based on the perfectly flexible and quasi-inextensible membrane theory, and its formulation does not rely on any tire material property - it is exclusively geometric.

Some of calculated results from this model were compared with measured data from four quite different types of tires, showing quite good approximation in all cases.

This formulation, assuming small displacements, is one additional step to a more comprehensive model of the tire dynamic behavior, which will be published later.
\end{abstract}

\section{INTRODUCTION}

This paper is a development of a previous article (SALVAGNI, R. B. 2015), and presents a mathematical model of some forces acting on tire-ground contact region. The basic concepts and assumptions were presented in that article, and they will not be repeated here. The forces associated to sidewall's shear deformations will be considered in another paper, to be published soon.

\section{PREVIOUS CONSIDERATIONS}

As seen before (SALVAGNI, R. B. 2015), concerning to the loads at the interaction tireground, the region of the tread in contact with the ground is isolated, and the corresponding force systems are applied to this region, building the so called "free body diagram" or "forces diagram" of the contact region. It is considered six distributed force systems acting in this region. The whole set of forces, in the absence of inertia forces, constitutes a null system, which implies the static equilibrium of this region. Five of these systems are shown in Figure 1.1 corresponding to the tire forces acting in the contact region. The sixtieth system, not shown, is the ground reaction on the contact region. From membrane hypothesis, this reaction system is equal and directly opposite to the combined other five ones. 


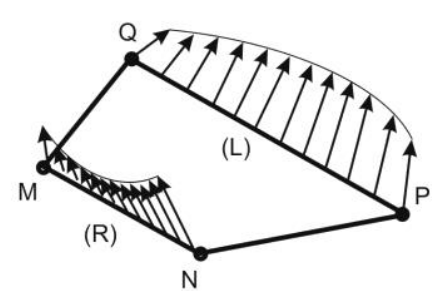

(a) Sidewalls force systems (two - right $(\mathrm{R})$ and left $(\mathrm{L}))$

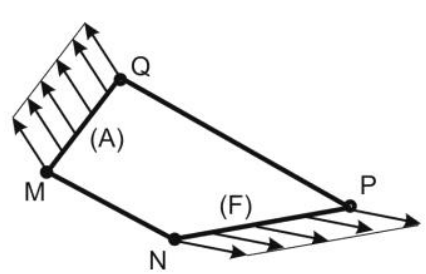

(b) Tread force systems (two - fore (F) and aft (A))

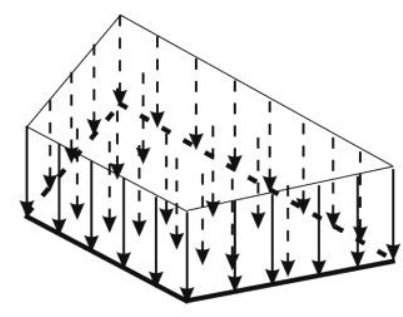

(c) Direct uniform pressure force system

Figure 1-1 - Force systems from the tire, at contact region

From basic mechanics, the system of all distributed forces applied to the contact region by the remaining of the tire, and its internal pressure, is equivalent to a system of one force and one moment $\left(\vec{R} t, \vec{M} t_{C}\right)$, where $\vec{R} t$ is the resultant of those forces, applied to a point $\mathrm{C}$ in the contact region, and $\vec{M} t_{C}$ is the binary equal to the moment of the same forces to pole $C$, as shown in Figure 1-2. Analogously, $\left(\vec{R} g, \vec{M} g_{C}\right)$ are the system equivalent to all the forces from the ground acting in this region. If we neglect dynamic effects, the force systems $\left(\vec{R} t, \vec{M} t_{C}\right)$ and $\left(\vec{R} g, \vec{M} g_{C}\right)$ are directly opposite and we have:

$$
\vec{R} t+\vec{R} g=\overrightarrow{0} \text { and } \vec{M} t_{C}+\vec{M} g_{C}=\overrightarrow{0}
$$

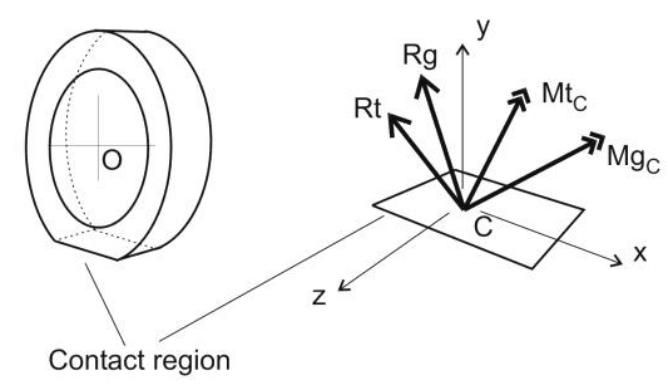

Figure 1-2 - Equivalent forces at contact region

Note that, for the steady state situation, we have also the relationship between the ground forces and the forces applied to the rim center $O$ :

$$
\vec{R} g+\vec{R}_{S}+\vec{R}_{A}=\overrightarrow{0} \text { and } \vec{M} g_{C}+\vec{M}_{S_{O}}+\vec{M}_{A_{O}}+(C-O) \times\left(\vec{R}_{S}+\vec{R}_{A}\right)=\overrightarrow{0}
$$

where $\left(\vec{R}_{S}, \vec{M}_{S_{O}}\right)$ are the forces corresponding to the initial static wheel load, and $\left(\vec{R}_{A}, \vec{M}_{A_{O}}\right)$ are the forces corresponding to the small relative displacement between the rim and the contact region, from the initial static position.

We have five force systems at the contact region, as shown in Figure 1-1. If we use $k$ to designate each of them:

$k=1$ : Right Left sidewall force system;

$k=2:$ Left sidewall force system;

$k=3$ : Fore tread force system;

$k=4:$ Aft tread force system;

$k=5:$ Direct pressure force system, 
Let us also represent the force system $\left(\vec{R} t, \vec{M} t_{O}\right)$, where $\vec{M}_{t_{O}}=\vec{M} t_{C}+(C-O) \times \vec{R}_{t}$, by a single vector $\{R\}$, with this five force systems:

$$
\{R\}=\left\{\begin{array}{c}
R t_{x} \\
R t_{y} \\
R t_{z} \\
M t_{O x} \\
M t_{O y} \\
M t_{O z}
\end{array}\right\}=\left\{\begin{array}{l}
F_{1} \\
F_{2} \\
F_{3} \\
F_{4} \\
F_{5} \\
F_{6}
\end{array}\right\}=\left\{R^{1}\right\}+\left\{R^{2}\right\}+\left\{R^{3}\right\}+\left\{R^{4}\right\}+\left\{R^{5}\right\}=\sum_{k=1}^{k=5}\left\{R^{k}\right\}
$$

where $F_{i}$ for $\mathrm{i}=1,2$ and 3 are the resultant force components in respectively $x, y$ and $z$ directions. Also, $F_{i}$ for $\mathrm{i}=4,5$ and 6 are the resultant moment components relative to the center of the wheel, about axis $x, y$ and $z$, respectively, and $\left\{R^{k}\right\}$ stands for the force system $k$.

\section{FORCES AND MOMENTS}

\section{$2.1 \quad$ Tire physical model}

The tire physical model was presented at previous article (SALVAGNI, R. B. 2015), with all hypothesis and simplifications. This model is represented in Figures 2.1 and 2.2.

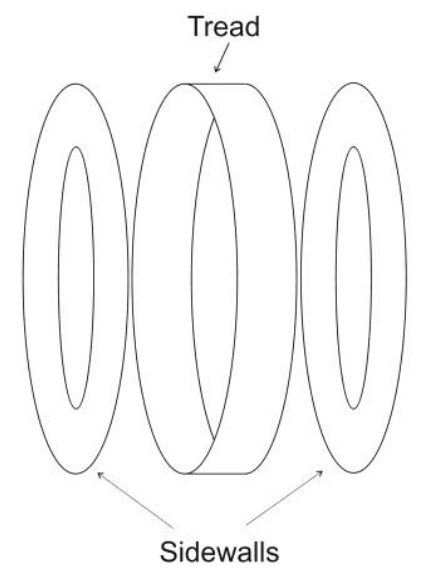

Figure 2-1 - Membrane model 


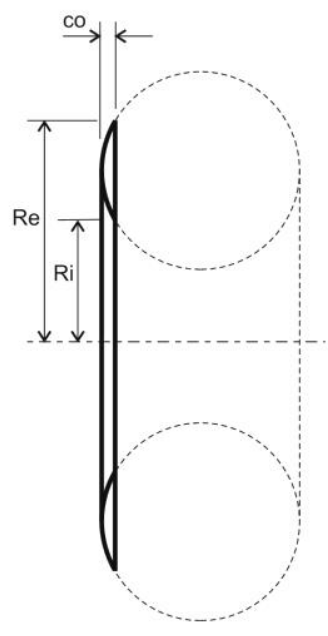

a. One Sidewall

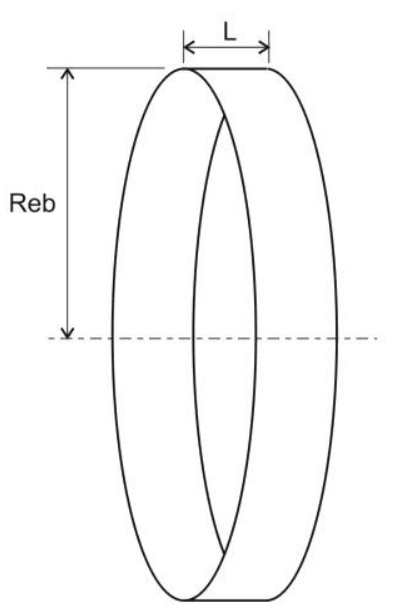

b. Tread

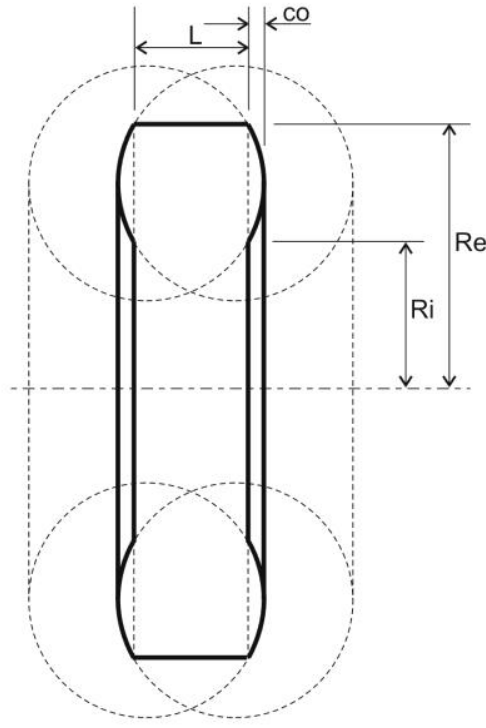

c. Membrane model

Figure 2-2 - Membrane model surfaces

\subsection{Sidewall forces system $(k=1$ and $k=2)$}

Let us consider the case (a) $(\mathrm{R})(\mathrm{k}=1)$ of Figure 1-1. The arc segment $\mathrm{AB}$, at the right sidewall, is shown in Figure 2-3. $O_{R}^{\prime}$ is the orthogonal projection of center $O$ ' to the right rim's plane.

The wheel and the tire tread have the same width $\mathrm{L}$ but, due the tire deformation, we will consider a small rotation $\xi$ about an axis parallel to $\overrightarrow{\mathrm{u}^{*}}$ passing by point $\mathrm{B}$.

Assume that the stress state of arch $A B$ is the same of a toroid surface with internal pressure $\mathrm{p}$ and dimensions defined from the parameters Re, Ri and c. Also, suppose that the stresses remain unchanged for small deformations of the sidewall. Then, in the plane of arch AB, the toroid radial stress is given by (YOUNG, et al., 2001):

$$
\sigma_{\mathrm{r}}=\mathrm{p} \rho_{0} / 2 \mathrm{~h}
$$

where $\mathrm{h}$ is the toroid wall thickness, $\mathrm{p}$ is the internal pressure and $\square 0$ is the no deformed sidewall's circumference radius corresponding to co, as shown on Figure 2-2. The distributed force, per unit length, applied by the right side sidewall to the contact region, acting at $\mathrm{B}$, is:

$$
\overrightarrow{\mathrm{f}}=-\sigma_{\mathrm{r}} \mathrm{h} \cdot \overrightarrow{\mathrm{q}}=-\mathrm{p} \rho_{0} / 2 \cdot \overrightarrow{\mathrm{q}}
$$

where $\vec{q}$ is the unit vector shown in Figure 2-3b. 


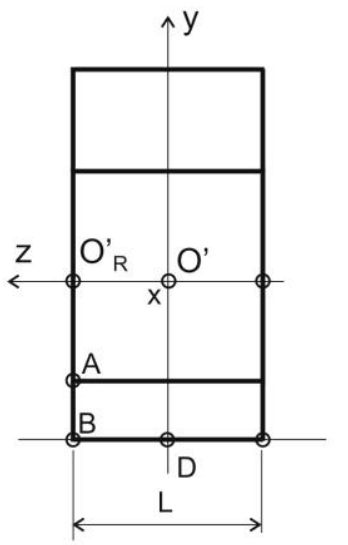

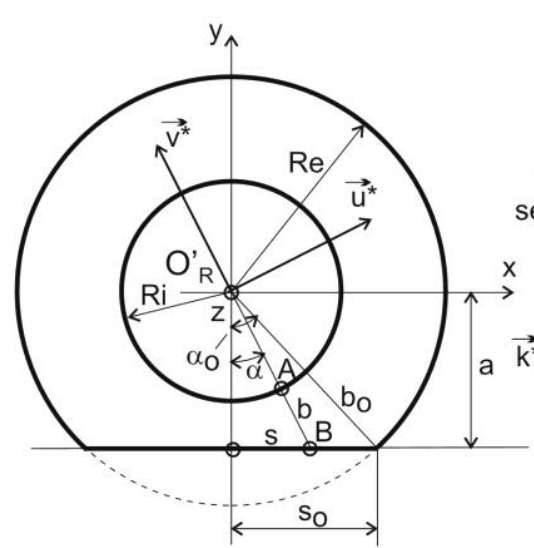

a. Tire

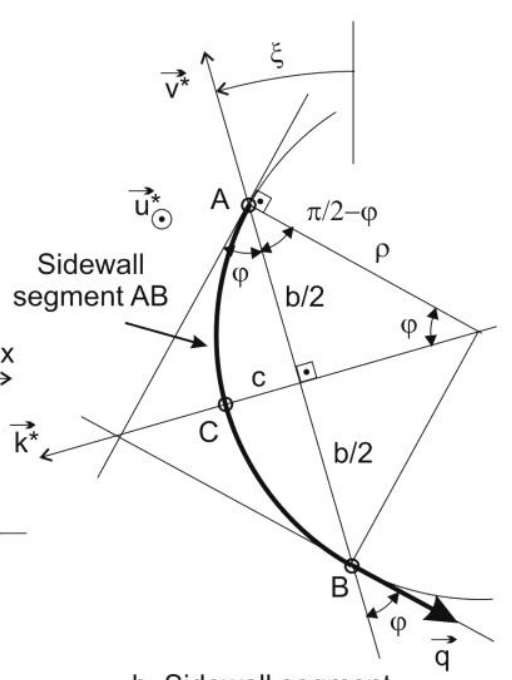

b. Sidewall segment

Figure 2-3 - Sidewall - dimensions and coordinates systems

The other toroid stress component, orthogonal to the plane of the arch $\mathrm{AB}$, selfequilibrates when the $\mathrm{AB}$ segment is considered and, therefore, is equivalent to zero in this segment.

The length $b o=\mathrm{Re}-\mathrm{Ri}$ and the parameter co correspond to the sidewall segment region out of the contact region; $\mathrm{b}$ and $\mathrm{c}$ correspond to the deformed sidewall region, along to the contact region.

Considering Figure 2-3, for a small angle $\xi$, the distributed force is:

$$
\begin{aligned}
\overrightarrow{\mathrm{f}}_{\mathrm{S}, \mathrm{R}}=\frac{\mathrm{p} \rho_{0}}{2}[( & -\cos \varphi \sin \alpha+\xi \sin \varphi \sin \alpha) \overrightarrow{\mathrm{\imath}}+(\cos \varphi \cos \alpha-\xi \sin \varphi \cos \alpha) \vec{\jmath} \\
& +(\xi \cos \varphi+\sin \varphi) \overrightarrow{\mathrm{k}}]
\end{aligned}
$$

The distributed force from equation (2-3) acting on the side $\mathrm{MN}$ of the trapezoidal contact region, as shown in Figure 2-4, is equivalent to a resultant $\overrightarrow{\mathrm{F}}_{S, R}$, applied to the point $\overline{\mathrm{S}}$ of the segment $\mathrm{MN}$, and a moment $\overrightarrow{\mathrm{M}}_{\mathrm{SR}, \overline{\mathrm{S}}}$ about the same point (pole) $\overline{\mathrm{S}}$. Let us define the oriented axis s, defined by segment $\mathrm{MN}$ and with origin in $\overline{\mathrm{S}}$.

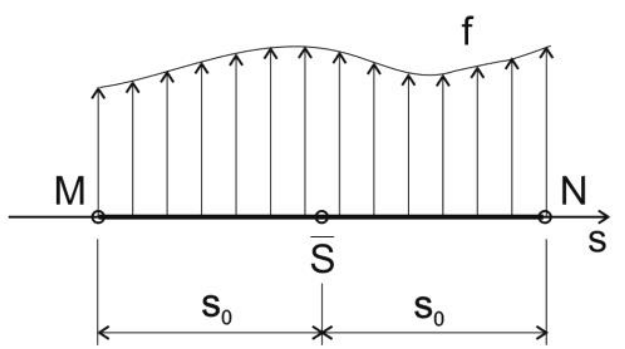

Figure 2-4 - Distributed force and s axis - sidewall

Thus, considering the symmetry, the resultant is: 


$$
\begin{gathered}
\overrightarrow{\mathrm{F}}_{\mathrm{SR}}(\mathrm{a}, \xi)=\int_{\mathrm{sM}}^{\mathrm{sN}} \overrightarrow{\mathrm{f}}_{\mathrm{S}, \mathrm{R}} \mathrm{ds}= \\
=\frac{\mathrm{p} \rho_{0}}{2} \int_{-\mathrm{so}}^{\mathrm{so}}[(-\cos \varphi \sin \alpha+\xi \sin \varphi \sin \alpha) \overrightarrow{\mathrm{\imath}} \\
+(\cos \varphi \cos \alpha-\xi \sin \varphi \cos \alpha) \overrightarrow{\mathrm{j}}+(\xi \cos \varphi+\sin \varphi) \overrightarrow{\mathrm{k}}] \mathrm{ds}
\end{gathered}
$$

The moment with respect to pole $\overline{\mathrm{S}}$, considering the symmetry, is:

$\overrightarrow{\mathrm{M}}_{\mathrm{SR}, \overline{\mathrm{S}}}=\int_{-\mathrm{so}}^{\mathrm{so}} \mathrm{s} \overrightarrow{\mathrm{\imath}} \times \overrightarrow{\mathrm{f}}_{\mathrm{S}, \mathrm{R}} \mathrm{ds}=-\frac{\mathrm{p} \rho_{0}}{2}\left[\int_{-\mathrm{so}}^{\mathrm{so}} \mathrm{s} \xi \cos \varphi \mathrm{ds} \vec{\jmath}+\int_{-\mathrm{so}}^{\mathrm{so}} \mathrm{s} \xi \sin \varphi \cos \alpha \mathrm{ds} \overrightarrow{\mathrm{k}}\right]$

For the case (a) $(\mathrm{L})(\mathrm{k}=2)$ of Figure 1-1, the procedure is analogous and we have:

$$
\begin{aligned}
& \overrightarrow{\mathrm{F}}_{\mathrm{SL}}(\mathrm{a}, \xi)=\frac{\mathrm{p} \rho_{0}}{2} \int_{- \text {so }}^{\mathrm{so}}[(-\cos \varphi \sin \alpha-\xi \sin \varphi \sin \alpha) \vec{\imath} \\
& +(\cos \varphi \cos \alpha+\xi \sin \varphi \cos \alpha) \vec{\jmath}+(\xi \cos \varphi-\sin \varphi) \overrightarrow{\mathrm{k}}] \mathrm{ds}
\end{aligned}
$$

and

$$
\overrightarrow{\mathrm{M}}_{\mathrm{SL}, \overline{\mathrm{S}}}=\frac{\mathrm{p} \rho_{0}}{2}\left[-\int_{-\mathrm{so}}^{\mathrm{so}} \mathrm{s} \xi \cos \varphi \mathrm{ds} \vec{\jmath}+\int_{-\mathrm{so}}^{\mathrm{so}} \mathrm{s} \xi \sin \varphi \cos \alpha \mathrm{ds} \overrightarrow{\mathrm{k}}\right]
$$

Then, for equation (1-3), we have:

and

$$
\left\{R^{1}\right\}_{\bar{S} R}=\frac{p \rho_{0}}{2}\left\{\begin{array}{c}
\int_{-s o}^{s o}(-\cos \varphi \sin \alpha+\xi \sin \varphi \sin \alpha) d s \\
\int_{-s o}^{s o}(\cos \varphi \cos \alpha-\xi \sin \varphi \cos \alpha) d s \\
\int_{-s o}^{s o}(\xi \cos \varphi+\sin \varphi) d s \\
-\int_{-s o}^{s o} s \xi \cos \varphi d s \\
-\int_{-s o}^{s o} s \xi \sin \varphi \cos \alpha d s
\end{array}\right\}
$$

$$
\left\{R^{2}\right\}_{\bar{s} L}=\frac{p \rho_{0}}{2}\left\{\begin{array}{c}
\int_{-s o}^{s o}(-\cos \varphi \sin \alpha-\xi \sin \varphi \sin \alpha) d s \\
\int_{-s o}^{s o}(\cos \varphi \cos \alpha+\xi \sin \varphi \cos \alpha) d s \\
\int_{-s o}^{s o}(\xi \cos \varphi-\sin \varphi) d s \\
-\int_{-s o}^{s o} s \xi \cos \varphi d s \\
\int_{-s o}^{s o} s \xi \sin \varphi \cos \alpha d s
\end{array}\right\}
$$

\subsection{Tire tread forces $(\mathrm{k}=3$ and $\mathrm{k}=4)$}


Let us consider the case (b) (F) of Figure 1-1.

Suppose a cylinder with radius Re and internal pressure $\mathrm{p}^{*}$. We define this pressure $\mathrm{p}^{*}$ as the sum of the internal gas pressure p plus the "equivalent pressure" p', which is the radial component of the loads applied by both sidewalls on the tire tread. This $\mathrm{p}$ ' is obtained from the $\overrightarrow{v^{*}}$ component of the distributed force $\vec{f}$ shown in equation (2-2), for $\varphi=\varphi 0$, corresponding to $\alpha=\alpha 0$ in Figure 2-5, were $\mathrm{L}$ is the width of the tire tread:

$$
\mathrm{p}^{\prime}=-\mathrm{p} \rho_{0} \cos \varphi_{0} / \mathrm{L} \Rightarrow \mathrm{p}^{*}=\mathrm{p} \cdot\left(\mathrm{L}-\rho_{0} \cos \varphi_{0}\right) / \mathrm{L}
$$

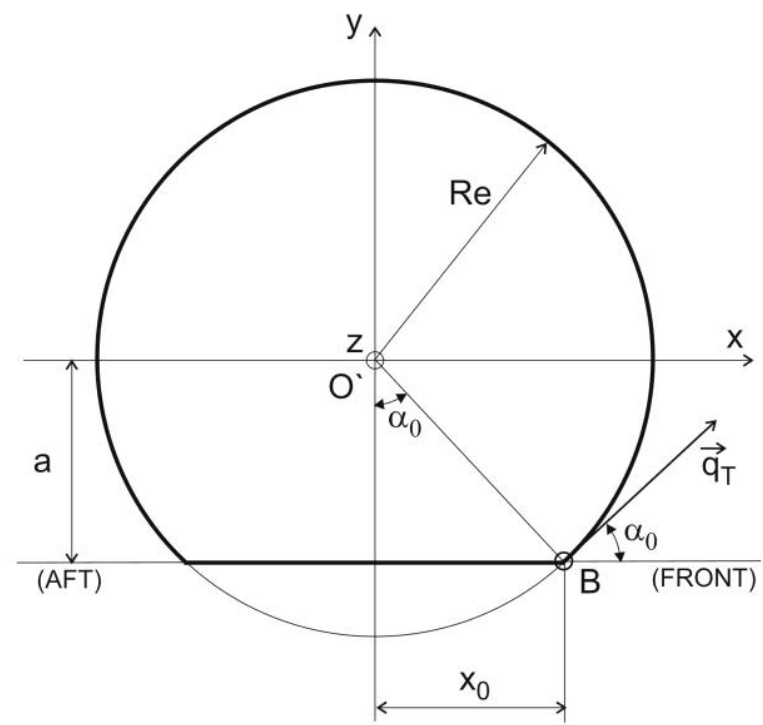

Figure 2-5 - Tire tread

The tension force by unit length, applied to the contact region by the tire tread, will be given by (YOUNG, et al., 2001):

$$
\overrightarrow{\mathrm{f}}_{\mathrm{T}}=\mathrm{p}^{*} \cdot \operatorname{Re} \cdot \overrightarrow{\mathrm{q}}_{\mathrm{T}} \cdot \frac{\mathrm{L}}{\left(\frac{\mathrm{L}}{\cos \theta}\right)}=\mathrm{p}^{*} \cdot \mathrm{Re} \cdot \overrightarrow{\mathrm{q}}_{\mathrm{C}} \cdot \cos \theta
$$

with $\theta$ shown in Figure 2-6. Neglecting the variation of $\alpha$ along the width $L$ and assuming that the forces remain parallel to plane $x y$, we have:

$$
\overrightarrow{\mathrm{q}}_{\mathrm{T}}=\cos \alpha_{0} \overrightarrow{\mathrm{l}}+\sin \alpha_{0} \overrightarrow{\mathrm{j}}
$$

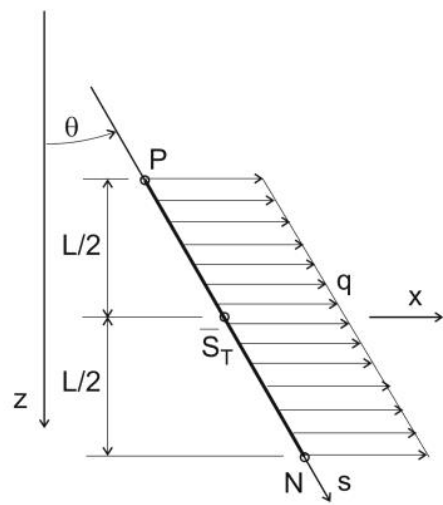


Figure 2-6 - Distributed forces and s axis - front tire tread

Defining the s axis as shown in Figure 2-6, we obtain the equivalent force system for the fore tire tread:

$$
\overrightarrow{\mathrm{F}}_{\mathrm{TF}}(\mathrm{a})=\int_{\mathrm{sN}}^{\mathrm{sP}} \overrightarrow{\mathrm{f}}_{\mathrm{T}} \mathrm{ds}=\mathrm{p}\left(\mathrm{L}-\frac{\mathrm{b}_{0}}{2 \tan \varphi_{0}}\right)\left(\mathrm{a} \vec{\imath}+\sqrt{\operatorname{Re}^{2}-\mathrm{a}^{2}} \vec{\jmath}\right)
$$

For the aft tire tread, analogously we have:

$$
\overrightarrow{\mathrm{F}}_{\mathrm{TA}}(\mathrm{a})=\mathrm{p}\left(\mathrm{L}-\frac{\mathrm{b}_{0}}{2 \tan \varphi_{0}}\right)\left(-\mathrm{a} \vec{\imath}+\sqrt{\mathrm{Re}^{2}-\mathrm{a}^{2}} \overrightarrow{\mathrm{\jmath}}\right)
$$

The moment about pole $\overline{\mathrm{S}}_{\mathrm{T}}$ is:

$$
\begin{gathered}
\overrightarrow{\mathrm{M}}_{\mathrm{TF}, \overline{\mathrm{S}}_{\mathrm{T}}}(\mathrm{a})=\int_{\mathrm{sN}}^{\mathrm{sP}} \mathrm{s} \cdot \overrightarrow{\mathrm{k}} \times \overrightarrow{\mathrm{f}}_{\mathrm{T}} \mathrm{ds}=\mathrm{p}^{*} \cdot \mathrm{Re} \cdot \overrightarrow{\mathrm{k}} \times \int_{\mathrm{sN}}^{\mathrm{sP}} \mathrm{s} \cdot \overrightarrow{\mathrm{q}}_{\mathrm{T}} \mathrm{ds}=\overrightarrow{0} \Rightarrow \\
\Rightarrow \overrightarrow{\mathrm{M}}_{\mathrm{TF}, \overline{\mathrm{S}}_{\mathrm{T}}}(\mathrm{a})=\overrightarrow{\mathrm{M}}_{\mathrm{TA}, \overline{\mathrm{S}}_{\mathrm{T}}}(\mathrm{a})=\overrightarrow{0}
\end{gathered}
$$

Then, for equation (1-3), we have:

and

$$
\left\{R^{3}\right\}_{\bar{S}_{T} F}=p\left(L-\frac{b_{0}}{2 \tan \varphi_{0}}\right)\left\{\begin{array}{c}
\sqrt{R e^{2}-a^{2}} \\
0 \\
0 \\
0 \\
0
\end{array}\right\}
$$

$$
\left\{R^{4}\right\}_{\bar{S}_{T} A}=p\left(L-\frac{b_{0}}{2 \tan \varphi_{0}}\right)\left\{\begin{array}{c}
\sqrt{R e^{2}-a^{2}} \\
0 \\
0 \\
0 \\
0
\end{array}\right\}
$$

\subsection{Direct pressure region}

This is the fifth force system applied on the contact region, corresponding to distributed pressure $p$ shown in Figure1-1(c).

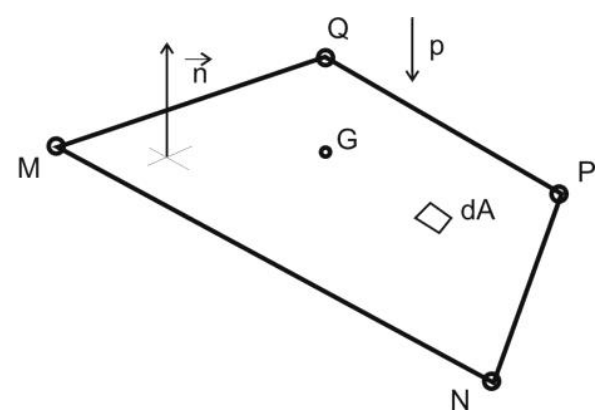

Figure 2-7 - Contact region under direct pressure

We will approximate the shape of this region by an isosceles trapezoid, observing that it is a rectangle if the relative displacement of the wheel to the contact region is null. Defining 
$\overrightarrow{\mathrm{n}}$ as the unit vector normal and positive upward to the contact surface, dA as the area element, and G as the trapezoid centroid, shown on Figure 2-7, we obtain the distributed force per unit area in the contact region (the trapezoid MNPQ):

$$
\overrightarrow{\mathrm{f}}_{\mathrm{p}}=-\mathrm{p} \cdot \overrightarrow{\mathrm{n}}
$$

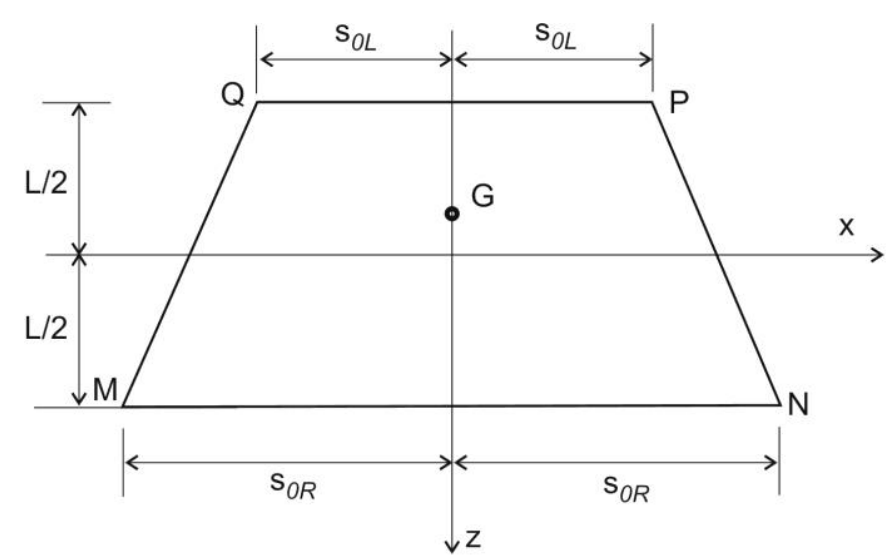

Figure 2-8 - Contact region as an isosceles trapezoid

Integrating by the whole area A of the trapezoid, as in Figure 2-8, we obtain the resultant:

where

$$
\overrightarrow{\mathrm{F}}_{\mathrm{p}}\left(\mathrm{a}_{\mathrm{R}}, \mathrm{a}_{\mathrm{L}}\right)=\int_{\mathrm{A}} \overrightarrow{\mathrm{f}}_{\mathrm{p}} \mathrm{dA}=-\mathrm{p} \overrightarrow{\mathrm{n}} \int_{\mathrm{A}} \mathrm{dA}=-\mathrm{pA} \overrightarrow{\mathrm{n}}=-\mathrm{pA} \vec{\jmath}
$$

$$
\begin{gathered}
A=\frac{L\left(s_{0 R}+s_{0 L}\right)}{2}=A_{R}+A_{L} \\
A_{R}=\frac{L}{2} s_{0 R} \\
A_{L}=\frac{L}{2} s_{0 L} \\
s_{0 R}=\sqrt{R^{2}-a_{R}^{2}} \\
s_{0 L}=\sqrt{R e^{2}-a_{L}^{2}}
\end{gathered}
$$

and $\mathrm{a}_{\mathrm{R}}$ and $\mathrm{a}_{\mathrm{L}}$ are the values of parameter a in the right and left sidewalls, respectively.

The center $\mathrm{G}$ of the uniform parallel forces has the coordinates:

$$
\begin{gathered}
\mathrm{x}_{\mathrm{G}}=0 \\
\mathrm{y}_{\mathrm{G}}=-\mathrm{a} \\
\mathrm{z}_{\mathrm{G}}=\frac{\mathrm{L}}{6}\left(\frac{\mathrm{s}_{0 \mathrm{R}}-\mathrm{s}_{0 \mathrm{~L}}}{\mathrm{~s}_{\mathrm{OR}}+\mathrm{s}_{0 \mathrm{~L}}}\right)
\end{gathered}
$$

The moment for pole $\mathrm{G}$, as we have a system of uniform parallel forces, is:

$$
\vec{M}_{p, G}\left(a_{R}, a_{L}\right)=\overrightarrow{0}
$$


Then, for equation (1-3), we have:

$$
\left\{R^{5}\right\}_{G}=-p A\left\{\begin{array}{l}
0 \\
1 \\
0 \\
0 \\
0 \\
0
\end{array}\right\}
$$

\section{COMPARISON WITH MEASURED DATA}

In a prior paper (SALVAGNI et all, 2013), the formulation presented here, for the case of vertical loads, was compared with measured values of three different tire types commonly used in passenger cars: tire for small passenger cars, high performance tire and tire used in SUV vehicles. In most cases, the differences were smaller than $5 \%$ at extreme values, and less than $1 \%$ at usual values for the normal vehicle's application.

The tables below show the results, and more details may be found in the original paper.

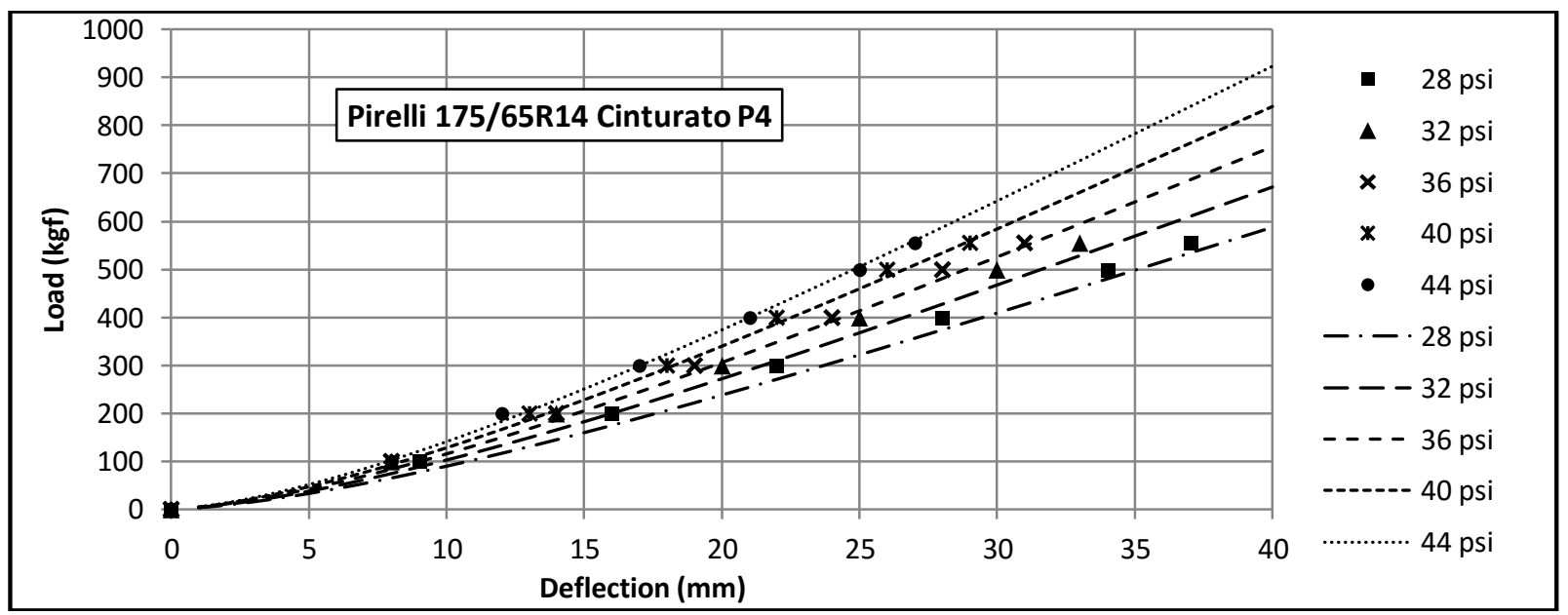

Figure 3-1 - Measured (points) x calculated (lines) vertical deflection - Tire: Pirelli 175/65R14 Cinturato P4 (Measured data by Pirelli Pneus Brasil)

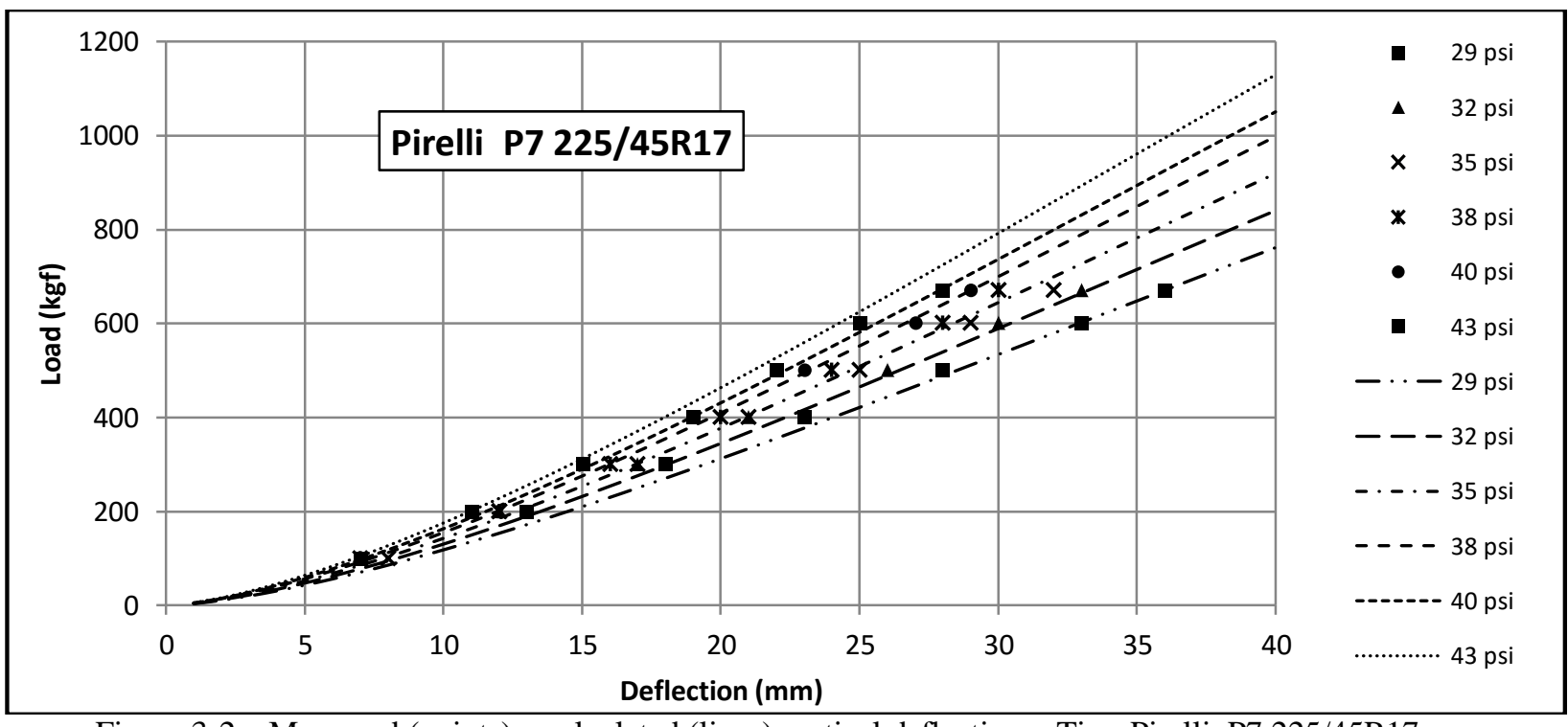

Figure 3-2 - Measured (points) x calculated (lines) vertical deflection - Tire: Pirelli P7 225/45R17 


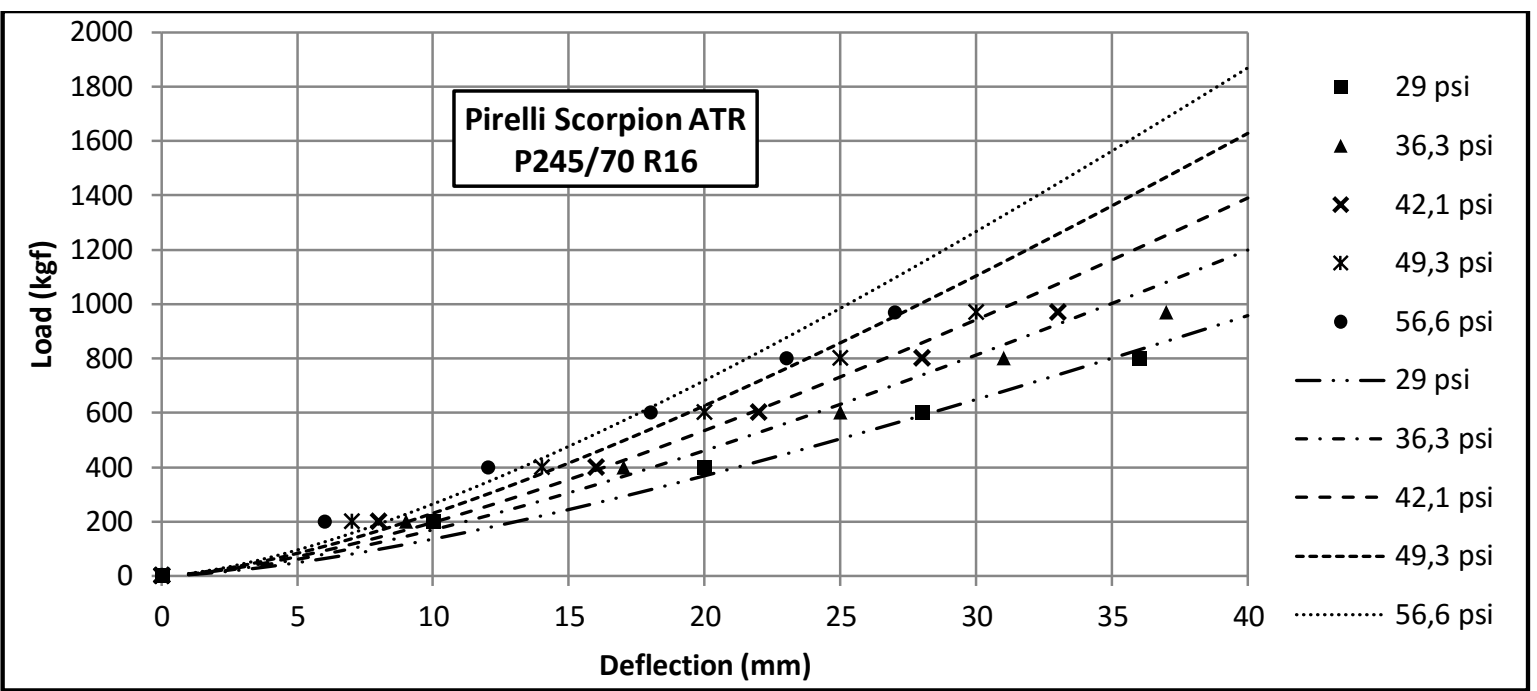

Figure 3-3 - Measured (points) x calculated (lines) vertical deflection - Tire: Pirelli Scorpion ATR P245/70 R16 (Measured data by Pirelli Pneus Brasil)

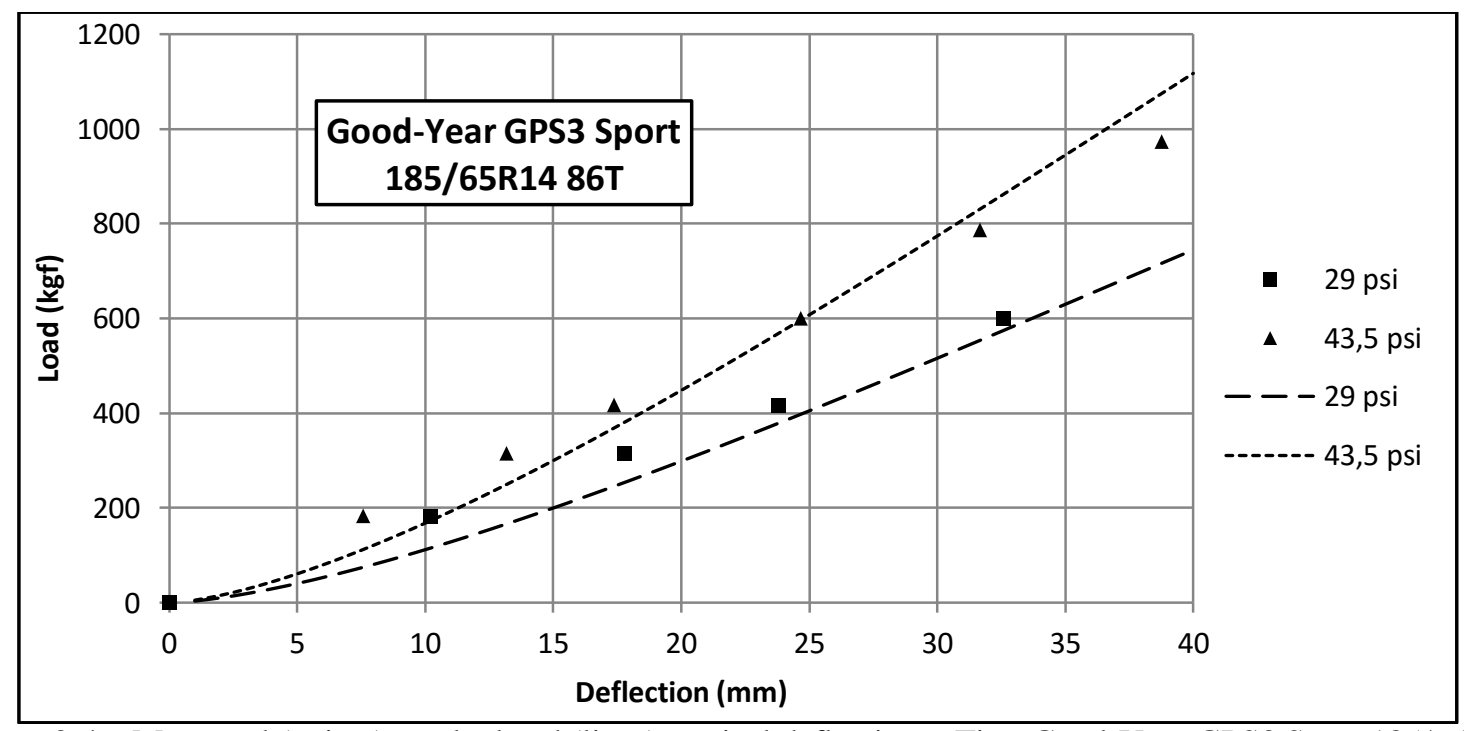

Figure 3-4 - Measured (points) x calculated (lines) vertical deflection - Tire: Good-Year GPS3 Sport 185/65R14 86T (Measured data by Volkswagen do Brasil)

\section{CONCLUSIONS}

This text presented a mathematical model for the forces on tire-ground contact region, based in a physical model stablished before (SALVAGNI, R. B. 2015). Here, these forces depend uniquely on the tire internal pressure, the tire geometry and the relative displacement between the rim and the contact region.

A partial comparison with measured data showed very good results.

The forces related to the shear deformation of sidewalls were not included here, and they will be considered in a further article. The next step is to make explicit the relationship between 
the forces and the relative displacements rim-contact region, which will be done in a next paper.

\section{REFERENCES}

SALVAGNI, R. B. 2015. Some Considerations About Forces and Deformations in Tires, p. 169-180 . In: In Anais do XXIII Simpósio Internacional de Engenharia Automotiva SIMEA 2014. São Paulo: Blucher, 2015. ISSN 2357-7592 (DOI 10.5151/engprosimea2015-PAP145).

SALVAGNI, R. B.; ALVES M. A. L.; BARBOSA R. S. 2013. "A GEOMETRICAL MODEL FOR TIRES UNDER VERTICAL LOADING", p.1-15. In XXI Simpósio Internacional de Engenharia Automotiva - SIMEA 2013. São Paulo: Blucher, 2014.(DOI: http://doi.org/10.5151/engpro-simea-PAP2)

YOUNG, W. and BUDYNAS, R. 2001. Roark's Formulas for Stress and Strain. 7th. s.1. : McGraw-Hill, 2001. 